\title{
Problem Solving and Finding Solutions for Children with Autism Spectrum Disorder: A Model
}

\author{
Ida Chauvin* and Walter Buboltz \\ Louisiana Tech University, USA \\ *Corresponding author: Ida Chauvin, Department of Psychology and Behavioral Sciences, Louisiana Tech University, PO Box \\ 10048, Ruston, LA 71272, USA
}

To Cite This Article: Ida Chauvin, Walter Buboltz. Problem Solving and Finding Solutions for Children with Autism Spectrum Disorder: A Model. Am J Biomed Sci \& Res. 2019 - 2(1). AJBSR.MS.ID.000569. DOI: 10.34297/AJBSR.2019.02.000569

Received: March 18, 2019 | Published: March 26, 2019

\section{Opinion}

Problem Solving and Finding Solutions for Children with Autism Spectrum Disorder: A Model Individuals with an Autism Spectrum Disorder (ASD) typically have higher support needs for life skills, social skills and mental health issues than most other populations and the amount of support needed likely will depend on the severity of the disorder and specific difficulties. In order to ensure high quality services for ASD individuals, a well-trained staff is essential for promoting life skills and decreasing mental health issues. The efficacy of essential personnel, as with all mental health issues, is hampered by the lack of formal and informal education and training, preparation and experience when working with ASD individuals. Not only is there a dearth of studies that present valid and reliable specific training for the treatment of ASD individuals, the few studies that do exist only offer modest information about effective strategies to intervene. Most do, however, indicate the need for understanding issues related to autism spectrum disorders and improving staff training with regard to the ASD population [1].

Thus, one of the major problems for assisting individuals with an ASD, is the lack of adequate training and understanding on the part of staff members. Some preparatory training for teachers, administrators, and counsellors/therapists should address the unique issues of the autism population as a whole while also incorporating other areas, such as diversity, parenting issues, individuals with disabilities, and ethical treatments of diverse populations. However, most training programs offer limited exposure and no in-depth preparation for staff member to work with this unique population. At the current time there are few published studies that detail effective approaches for educators and service providers that can be applied to the autism spectrum disorder population.

The number of individuals diagnosed with Autism Spectrum Disorder (ASD) is on the rise. Research indicates the prevalence of ASD is 1 in 68 of all age groups and the number of children diagnosed with an ASD is even higher. In the United States the prevalence may be as high as $2 \%$ of the population (Center for Disease Control, 2015) [2]. At the current time no clear information or reason for the rise in ASD diagnosis has been isolated. Whether this trend will continue is unknown.

ASD is diagnosed, according to the Diagnostic and Statistical Manual-V (DSM-V, 2013), as pervasive difficulties in communication and social interaction, as well as restricted interests and inflexibility in behaviours that are observed from an early age and cannot be account for better by another disorder. The accepted practice for determining a diagnosis is a for detailed assessment that examines all the major areas of autism and determining if the individual exhibits all the aspects associated with an ASD or only some of the aspects associated with an ASD. Individuals who exhibit symptoms across all areas are typically considered "Autistic", while individuals exhibiting symptoms in many areas, but not all areas or have a decreased level of symptomology in many areas are consider having an Autism Spectrum Disorder.

Research and experience indicate that individuals living with autism or an autism spectrum disorder can lead a full life in spite of their associated challenges in the community and their families. By have assistance in adapting and adjusting to their lifestyle, developing adaptive skills and learning appropriate social skills increase the change of living a fulfilling life particularly when early interventions are in place. Positive interactions and the instillation of hope appears to be extremely important and the development of hope and interactions is related to the best possible outcomes. Without hope, situations involving psychological stress can deteriorate rapidly and possibly lead to further difficulties.

Fostering support and hope with both parents and service providers may be the most valuable contributions one can make in working with and supporting individuals with an ASD. Best practices indicate a critical need for families, all teachers involved, therapists and staff to coordinate efforts in order to produce the best outcomes. These team members all need to be on the same page of treatment 
to support and ensure that there is open communication, allowing them to work collaboratively to ensure the best possible outcomes. Collaboration is the key. For example, working on different goals at home and school may lead to confusion on the part of the ASD individual or lead to decreased hope as progress is not reinforced in all situations. Both in the United States and abroad, it is noted that the gold-standard for treating ASD individuals is with training in the science of applied behaviour analysis (ABA).

These services are not readily available in all areas. In rural areas, there is a severe shortage of ABA services. In lieu of ABA, schools and agencies are scrambling to find interventions to assist in providing services for ASD individuals. Although IDEA'97 required school districts to conduct functional behavioural assessments for students with behaviours negatively affecting learning, the application of behavioural models in the schools is still in its infancy [3]. Clearly, there is a need for progress even today.

It is of great concern that it is estimated that during initial training of specialties such as nurses, speech and language therapist, occupational therapists, psychologists and other medical and allied health providers have limited training in this area. Most will have received only a maximum of one to 3 hours of academic training related to behavioral analysis or treatment of individuals with an ASD and other developmental difficulties. Social workers and teachers may actually receive no training in working with an ASD or developmental disabilities at all [4].

In view of this lack of training and service availability, collaboration and coordination between families and serviceproviders can have a substantial impact on the success of an ASD individual, the families and the service providers. A model of collaborative problem solving is recommended for improving services for individuals with an ASD and other developmental difficulties. There is a dearth of information about communication between families and providers but the literature that is available show a considerable impact on meeting the needs, changing behaviors, and solving mutual concerns of families and providers when coordination and collaboration is present between all parties involved.

Problem solving methods need not be complicated. Tilly [5,6] recommends four general thematic questions that are the basis for various problem-solving models: a.) what is the problem? (a clear definition of the problem), b.) why is it occurring? (possible causes or what is maintaining the defined problem at this time) c.) What are possible solutions? (Select an intervention and noting the specifics (the "who, what, when and where" of the solution.) d.) What are the outcomes? If concerned parties such as parents, teachers and other professionals share a mutual concern, have a clearly defined problem, try to determine what possibly could be the cause or maintaining the problem, and mutually design and perform an intervention to correct the problem, better outcomes can be expected. Through this process all parties involved can openly communicate, collaborate, and focus on the same problem, they will be able to share information about the outcomes and effectiveness of the plan and make alternations needed to ensure the best possible outcomes.

Alliances formed between the various parties, such as the teacher and the parents, will avoid adversarial relationships that can damage the collaborative process and outcomes. Team approaches such as practices that begin at home and are reinforced at school and visa-versa have a better chance of success as all parties are working together and not against each other [7].

Lastly, intensive preservice preparation and professional development for all stake-holders who work with children with autism and other disabilities is necessary to ensure the necessary skills and knowledge to work with this population. To improve the academic achievement and functional performance of children with disabilities, the use of scientifically based instructional practices should introduced and utilized to the maximum extent possible.

\section{References}

1. American Psychological Association (APA) (2013) Diagnostic Statistical Manual (DSM-5). Am J Psychiatry.

2. Centers for Disease Control and Prevention (CDC) (2015) Autism Spectrum Disorder (ASD): Data and Statistics.

3. Pratt C, Buckman S (1999) Supporting students with Asperger's syndrome who present behavioral challenges. The Reporter 4(3): 6-10.

4. Dillenburger K, Röttgers HR, Dounavi K, Sparkman C, Keenan M, et al. (2014) Multidisciplinary teamwork in autism: Can one size fit all? Aust Educ Dev Psychol 30: 1-16.

5. Tilly WD (2008) Best practices in school psychology V. Citeseer.

6. Tilly WD (2002) Best Practices in School Psychology as a ProblemSolving Enterprise. A Thomas \& J Grimes (Eds.), Best Practices in School Psychology 4: 21-36.

7. National Association of School Psychologists, Washington, DC, USA. 